\title{
Simultaneous in situ detection of protein expression of multiple tumor markers of circulating tumor cells and heteroploid of chromosome 8 in primary lung cancer
}

\author{
Hanqing Yao ${ }^{1,2}$, Zhengdong Wang ${ }^{1}, J^{\text {Junjun }}$ Yang $^{1}$, Xingxiang $\mathrm{Xu}^{1}$, Yong Song ${ }^{2}$ \\ ${ }^{1}$ Department of Respiratory and Critical Care Medicine, Clinical Medical College, Yangzhou University, Yangzhou, China; ${ }^{2}$ Department of \\ Respiratory and Critical Care Medicine, Jinling Hospital, the First School of Clinical Medicine, Southern Medical University, Nanjing, China \\ Contributions: (I) Conception and design: H Yao, X Xu, Y Song; (II) Administrative support: X Xu, Y Song; (III) Provision of study materials or \\ patients: H Yao, Z Wang, J Yang; (IV) Collection and assembly of data: H Yao, Z Wang, J Yang; (V) Data analysis and interpretation: H Yao, Z Wang; \\ (VI) Manuscript writing: All authors; (VII) Final approval of manuscript: All authors. \\ Correspondence to: Yong Song. Department of Respiratory and Critical Care Medicine, Jinling Hospital, the First School of Clinical Medicine, \\ Southern Medical University, No. 305 Zhongshan East Road, Nanjing 210008, China. Email: yong.song6310@gmail.com; Xingxiang Xu. \\ Department of Respiratory and Critical Care Medicine, Clinical Medical College, Yangzhou University, No. 98, Nantong West Road, Yangzhou \\ 225001, China. Email: 18051062315@yzu.edu.cn.
}

Background: Previously published studies have shown that circulating tumor cells (CTC) play an important role in clinical staging, efficacy monitoring and prognostic evaluation of lung cancer. The aim of the present study was to investigate the significance of simultaneous in situ detection of multiple tumor marker protein expression and chromosome 8 aneuploid of CTC in the diagnosis and treatment of primary lung cancer.

Methods: We investigated the expression of cytokeratin 18 (CK18) and Vimentin on the surface of CTC and the aneuploidy of chromosome 8 in the peripheral blood of 24 patients with metastatic primary lung cancer, which were detected by subtraction enrichment (SE) and immunofluorescence in situ hybridization (iFISH). Their correlation with clinicopathological features, curative effect, and prognosis was analyzed.

Results: The positive rate of CTC was $95.83 \%$ (23/24). There was a certain correlation between the positive rate of CTC and smoking, and a correlation between the number of CTC and histopathological type. The number of monoploid, diploid, triploid, and polyploid CTC had no statistical correlation with progression-free survival (PFS) or overall survival (OS). However, a tetraploid CTC count of $\geq 2$ was an unfavorable predictor of response; a tetraploid CTC count $\geq 1$ was an unfavorable prognostic predictor of poor OS. The rate of CK18+ CTC in all 24 patients was $4.35 \%$ (1/23). Seven out of these 24 patients were also tested for Vimentin, and the rate of Vimentin+ CTC was 85.71\% (6/7). Small-cell $(\leq 5 \mu \mathrm{m})$ CTC was found in 6 of these 7 patients and accounted for $11.83 \%$ (11/93) of the total CTC. The tumor marker phenotype of small-cell CTC was CK18- and Vimentin+. In addition, circulating tumor microthrombi (CTM) were found in 2 of these 7 patients (28.57\%).

Conclusions: SE-iFISH has a high detection rate of CTC in the peripheral blood of patients with metastatic primary lung cancer, and it can identify small cell CTC. Tetraploid CTC count $\geq 2$ can predict poor PFS in patients with advanced lung cancer. Tetraploid CTC count $\geq 1$ could predict poor OS in patients with advanced lung cancer. CTM can predict poor prognosis in patients with lung cancer.

Keywords: Lung cancer; circulating tumor cells (CTC); chromosomes; aneuploidy; prognosis

Submitted Oct 22, 2021. Accepted for publication Dec 17, 2021.

doi: 10.21037/atm-21-6346

View this article at: https://dx.doi.org/10.21037/atm-21-6346 


\section{Introduction}

Lung cancer is a serious threat to human health, and its morbidity and mortality rank first among malignant tumors. The main treatments of lung cancer include surgery, chemotherapy, radiotherapy, molecular targeted therapy, anti-angiogenesis therapy, and immunotherapy. Sensitive and effective biomarkers during the course of treatment can evaluate the efficacy, monitor the progress of disease, and predict the prognosis of disease in real time, and can provide a basis for individualized and precise treatment of lung cancer.

At present, the evaluation of the curative effect of lung cancer is mainly based on imaging and serum tumor markers, but imaging has a certain lag, and the sensitivity and specificity of serum tumor markers are poor. For example, the sensitivity of carcinoembryonic antigen (CEA) in the diagnosis of lung cancer is only about $40 \%(1,2)$, and there are false negatives and false positives. CEA in the evaluation of efficacy and prognosis of lung cancer is still controversial.

With the emergence of "liquid biopsy" technology, circulating tumor cells (CTC) have become in lung cancer evaluation. CTC are malignant tumor cells that enter the circulating blood after shedding from a primary tumor or metastatic tumor, and have the characteristics of tumor genes or tumor antigens (3). CTC are mainly found in the blood of patients with malignant tumors. It is rare in the blood of patients with benign diseases and healthy people (4). Some CTCs with the characteristics of tumor stem cells proliferate and differentiate continually, which have become the root of tumor generation, development, and recurrence. Therefore, the existence of CTCs is the intermediate process of malignant tumors forming metastatic lesions at a distance. More and more studies show that CTC play an important role in the metastasis of lung cancer $(5,6)$. Researchers have found that CTC can form circulating tumor microthrombi (CTM; $\geq 2$ CTC), and have stronger viability and potential to form micrometastasis than single CTC; therefore, CTM are risk factors for tumor metastasis (7).

The detection method of CTC is mainly composed of 2 main links: separation and identification. The sensitivity and specificity of CTC detection are determined by the effectiveness of the separation and identification methods. The biggest difference between subtraction enrichment (SE) technology and the negative enrichment method is that SE technology uses special non-blood-derived cell separation medium to remove red blood cells instead of lysing red blood cells by hypotonicity. In the process of leukocyte elimination of 6-8.5 mL blood sample, a combinatorial population of multiple anti-leukocyte antibodies, rather than a single anti-CD45 antibody, is coupled to specially coated magnetic beads, thereby ensuring maximal specific removal of leukocytes and minimal non-specific adhesion to tumor cells. The CTC enriched by SE technology has no hypotonic damage and is not combined with the antibody, so it keeps good biological activity. The SE method does not depend on the expression of tumor cell surface markers; therefore, the tumor cells enriched by it are not limited by cell size and highly heterogeneous expression of tumor markers $(8,9)$. In 2009, it was reported for the first time that the application of SE technology could effectively detect CTC in $86 \%$ of recurrent lung cancer patients (10).

Chromosomal instability exists in tumor cells, and aneuploid cells can exist in both primary and metastatic lesions (11). According to reports, chromosome 8 aneuploidy in CTC is associated with progression-free survival (PFS) and overall survival (OS) in lung, breast, ovarian, and oral cancers (12-14). It has been extensively reported that chromosome 8 centromere probes (CEP 8) have been applied for the diagnosis of lung, esophageal, pancreatic, gastric, colorectal, bladder, and liver cancers $(6,15-20)$.

Immunofluorescence in situ hybridization (iFISH) is a novel detection method that combines immunofluorescence staining with fluorescence in situ hybridization (FISH), which can effectively detect non-hematogenous aneuploid CTC (21). The targeted non-hematogenous cells (CD45-) were detected for chromosomal aneuploidy by FISH and various tumor markers by immunofluorescence staining. Different from the conventional CK single-tumor-labeled fluorescence staining for identifying CTC, iFISH can be used on the basis of FISH, and furthermore, provide a dualcolor, double-tumor-labeled protein expression detection which can be freely selected [e.g., CK, epithelial cell adhesion molecule (EpCAM), human epidermal growth factor receptor-2 (HER-2), Vimentin, CD133, and CA19-9] and combined (e.g., CK+ EpCAM, HER-2+ EpCAM, and Vimentin+ EpCAM).

It has been demonstrated that EpCAM-independent iFISH can identify CTC with high sensitivity through surface marker expression and ploidy analysis (9), with a sensitivity of $92 \%$ in lung cancer (22). On this basis, combining with SE technology can results in the detection of more CTC, especially CK- CTC with epithelial- 
mesenchymal transition (EMT) (23). Benefiting from the advantages of SE technology combined with iFISH, we can identify and count the enriched tumor cells to explore their potential clinical value, especially in the evaluation of curative effect and prognosis of lung cancer.

Cytokeratin 18 (CK18) is a structural protein that is highly expressed in epithelial cells. It is a specific immunohistochemical marker of epithelial tumors, which is closely related to the type, proliferation and differentiation of epithelial cells. Vimentin is the most commonly used molecular marker of EMT, and its expression reflects the degree of EMT. Its number is positively correlated with the degree of malignancy and poor prognosis of tumors (24).

In the present study, we integrated the phenotypic analysis of CTC and karyotypic analysis of chromosome 8 , and analyzed the chromosome ploidy of CTC in the peripheral blood of patients with lung cancer based on different copy numbers of chromosome 8 . The expression of CK18 and Vimentin on the surface of tumor cells was detected independent of epithelial cell adhesion molecule (EpCAM). We investigated the significance of simultaneous in situ detection of multiple tumor marker protein expression and chromosome 8 aneuploid of CTC in the diagnosis and treatment of primary lung cancer.

We present the following article in accordance with the REMARK reporting checklist (available at https://dx.doi. org/10.21037/atm-21-6346).

\section{Methods}

\section{Clinical data and sample collection}

From July 1, 2017 to August 18, 2017, 24 patients with locally advanced or metastatic primary lung cancer (stage IIIB-IVB) were diagnosed by pathology at the Department of Respiratory and Critical Care Medicine of Northern Jiangsu People's Hospital. There were 16 males and 8 females. The age range was 42 to 77 years, with a median age of 65 years. Histopathological classification was as follows: 17 adenocarcinoma cases, 4 squamous cell carcinoma cases, and 3 small cell lung cancer cases. The study was conducted in accordance with the Declaration of Helsinki (as revised in 2013) and approved by the Institutional Ethical Committee of Northern Jiangsu People's Hospital (No. 2013010). All patients provided signed informed consent.

The following clinical data were collected: sex, age, smoking history, histopathological type, epidermal growth factor receptor (EGFR) gene mutation, Eastern Cooperative Oncology Group (ECOG) score, serum CEA, imaging data, first-line treatment, PFS, and OS. The clinical staging of non-small cell lung cancer (NSCLC) was performed according to Union for International Cancer Control/ American Joint Committee on Cancer (UICC/AJCC) 8th edition TNM staging system. Staging criteria for small cell lung cancer (SCLC) are established by the International Association for the Study of Lung Cancer (IASLC), and are divided into the limited stage and extensive stage.

According to the histological types and EGFR mutations, the first-line treatment was determined. Among 17 patients with adenocarcinoma, 4 patients with EGFR mutations were treated with EGFR tyrosine kinase inhibitor (EGFRi), and the remaining 13 cases were without EGFR mutations; 11 patients were treated with platinum-based chemotherapy (AP: pemetrexed + platinum or TP: paclitaxel + platinum) and 2 patients were not treated. All 4 squamous cell carcinoma patients without EGFR mutations were treated with platinum-based chemotherapy. EGFR was not detected in 3 patients with SCLC. Two cases were treated with EP (etoposide + platinum) regimen and 1 case was not treated. Peripheral blood samples were taken to detect serum CEA before treatment, and patients were followed up regularly to calculate PFS and OS (Table 1). RECIST version 1.1 was used to evaluate the efficacy in solid tumors for objective assessment of clinical response.

Peripheral venous blood $(6 \mathrm{~mL})$ from each patient was collected into an ethylene diamine tetraacetic acid (EDTA) anticoagulated tube and all samples were processed within 5 $\mathrm{h}$ of collection.

\section{Separation of CTC by SE technology}

A total of $6 \mathrm{~mL}$ of peripheral anticoagulant blood was centrifuged at $600 \times \mathrm{g}$ and separated. The supernatant fluid was discarded and $3 \mathrm{~mL}$ of Cytointelligen cell separation solution (Cytointelligen, China) was added, and centrifuged at $350 \times \mathrm{g}$ for $6 \mathrm{~min}$. After centrifugation, all the liquid on the bottom layer of the deposited red blood cells was transferred to a $50 \mathrm{~mL}$ centrifuge tube and slowly added to the magnetic beads buffer while shaking the centrifuge tube. Immunomagnetic beads of a mixture of anti-leukocyte antibodies (CK18, Vimentin) were added and incubated for $15 \mathrm{~min}$ at room temperature, after which they were placed on magnetic racks for magnetic separation. Aft $4 \mathrm{~min}$, the liquid in the centrifuge tube was carefully transferred to a new $15 \mathrm{~mL}$ centrifuge tube. Cleaning solution was added 
Table 1 Summary of serum CEA, first-line treatment plan and survival data of 24 selected patients

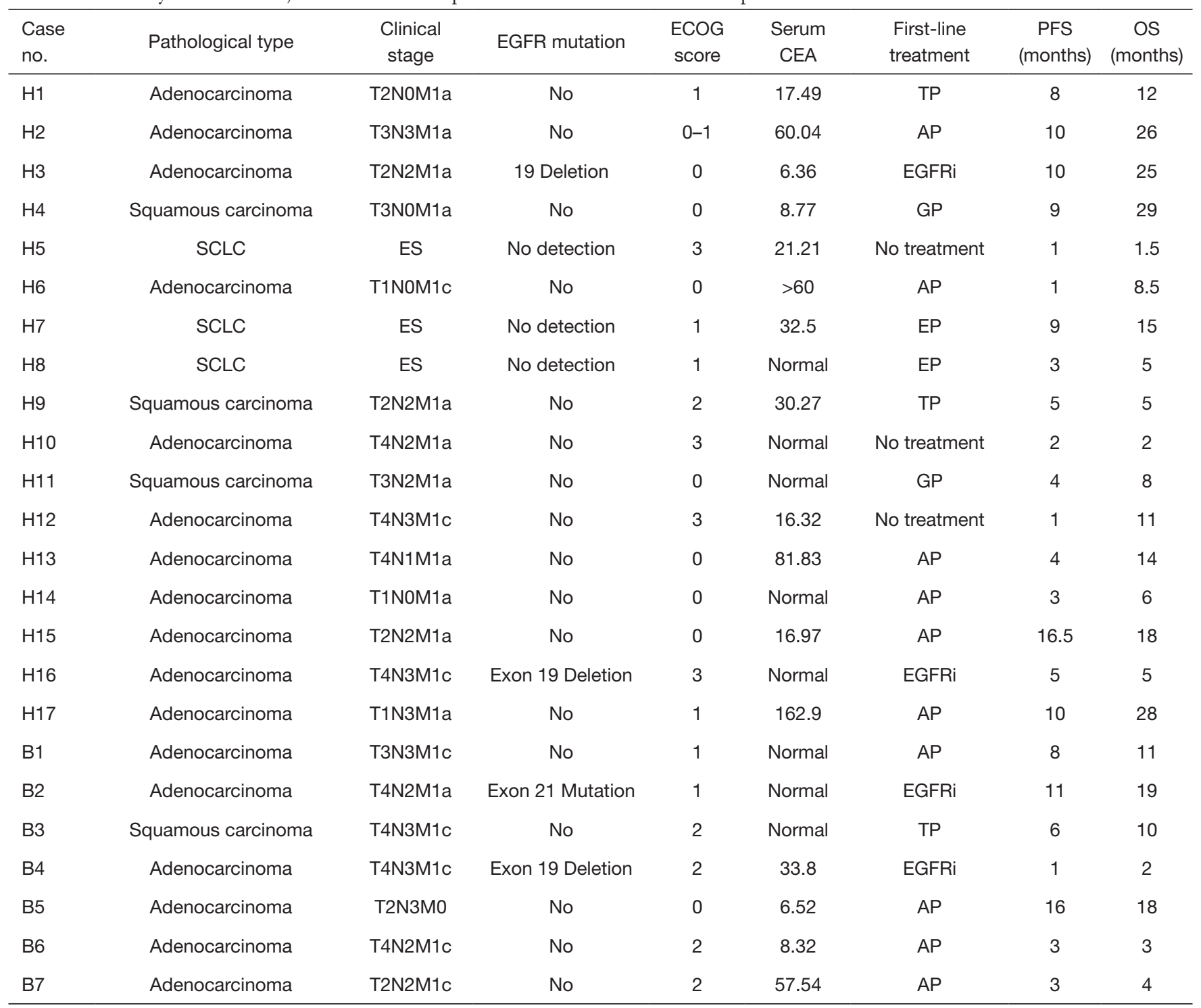

AP, pemetrexed + platinum; CEA, carcinoembryonic antigen; ECOG, Eastern Cooperative Oncology Group; EGFR, epidermal growth factor receptor; EP, etoposide + platinum; GP, gemcitabine + platinum; OS, overall survival; PFS, progression-free survival; EGFRi, EGFR tyrosine kinase inhibitor; TP, paclitaxel + platinum; SCLC, small cell lung cancer.

twice and centrifuged. The supernatant was discarded, and tissue fixing solution was added. The final pellet cells were then counted. The specimen solution was added to the specimen frame of the iFISH slide (Cytointelligen, China) (1 person/slide). Specimens were dried and stored for tumorlabeled iFISH.

\section{iFISH detection}

A mixed solution prepared by tissue fixing solution and the sample diluent was added to a CTC glass slide sample frame to cover the sample frame, and was left to stand for $10 \mathrm{~min}$ at room temperature in the dark. The slide was inserted into the ethanol jar and allowed to stand for $2 \mathrm{~min}$, and then taken out and dried. A total of $10 \mu \mathrm{L}$ of CEP 8 probe was added into the center of the specimen frame and covered with a cover glass to allow the liquid to spread to the whole specimen frame. The four edges of the cover glass was sealed with rubber cement, and directly put into a hybridization instrument for hybridization. Denaturation 
was at $76{ }^{\circ} \mathrm{C}$ for $10 \mathrm{~min}$ and hybridization was at $37{ }^{\circ} \mathrm{C}$ for $3 \mathrm{~h}$. The cover glass was washed off, and $10 \mu \mathrm{L}$ of DAPI staining solution for blood cell analysis was added dropwise to the glass specimen frame treated with the washing solution after drying. At last, cover glass was placed, sealed, and stored at $2-8{ }^{\circ} \mathrm{C}$ in the dark.

Images were observed under an Zeiss Axio Imager Z2 fluorescence microscope (Carl Zeiss AG, Germany), and the CTC images were scanned and analyzed automatically using a Metafer 5 system (Carl Zeiss AG, Germany).

\section{Identification of CTC}

CK18, Vimentin, CD45, DAPI, and CEP 8 staining data observed with fluorescence microscopy were combined to identify CTC. Immunostaining for CD45 was used to exclude hematogenous leukocytes and DAPI staining was used to observe the nucleus. CK18 and Vimentin are two tumor markers for CTC. To identify CTC subtypes, CEP 8 was used to count the copy number of chromosome 8 . CTC were classified as monosomic, triploid, tetraploid, and polyploid ( $\geq 5$ copies) based on the copy number of chromosome 8. CTC: DAPI+/CD45-/CK+-/Vimentin+-/ CEP 8+ (monosomic, triploid, tetraploid, polyploid, or diploid).

\section{Measurement of CEA}

Peripheral venous blood $(3 \mathrm{~mL})$ was collected into nonanticoagulant blood collection tubes (Becton Dickinson, Franklin Lakes, NJ, USA) and centrifugated (1,500 ×g) at room temperature for $10 \mathrm{~min}$. CEA was measured from the supernatant using the Cobas e601 automatic immunoassay analyzer (Roche, Pleasanton, CA, USA). The reference range of CEA was $<4.7 \mathrm{U} / \mathrm{mL}$.

\section{Efficacy evaluation criteria}

All patients received chemotherapy for 2 cycles (68 weeks) before the start of the third cycle of chemotherapy according to the "solid tumor efficacy evaluation criteria RECIST version 1.1" to evaluate the change of tumor size and clinical response objectively. Evaluation criteria were as follows: complete response, partial response, stable disease, and progressive disease.

Patients were followed up to December 31, 2019; 24 patients had relatively complete follow-up data. All variables were based on the medical records at the time of diagnosis.

\section{Statistical analysis}

Statistical analysis was performed using SPSS 26.0 software (IBM, Armonk, NY, USA); $\chi^{2}$ test was used to analyze the correlation between the positive rate of CTC and clinicopathological features. Mann-Whitney $U$ test and Kruskal-Wallis test were used to analyze the correlation between the number of CTC and clinicopathological features. Mann-Whitney $U$ test was used to analyze the correlation between CTC threshold of different ploidy of chromosome 8 and PFS or OS. $\mathrm{P}<0.05$ was considered statistically significant, and the $\mathrm{P}$ value was bilateral.

\section{Results}

\section{Correlation between positive rate of CTC in peripheral blood and clinical characteristic}

In the present study, peripheral blood tumor cells of 24 patients with primary lung cancer were detected by SEiFISH. The patients with No. B1-B7 used double-tumorlabeled CK18 and Vimentin 5-color channels. CTC were detected in 23 of the 24 patients, bringing the total number of detected CTC to 285. The average number of CTC per case was $12.4(285 / 23)$, and the positive rate of CTC was $95.83 \%$ (23/24). There was no significant correlation between the positive rate of CTC and the clinical characteristics of patients (sex, age, ECOG score, histological type, serum CEA), but there was a certain correlation between the positive rate of CTC and smoking status $(\mathrm{P}<0.05)$ (Table 2). There was no significant correlation between the number of CTC and clinical characteristics (sex, age, ECOG score, serum CEA) ( $\mathrm{P}>0.05)$, but there was a correlation between the number of CTC and histological type $(\mathrm{P}<0.05)$ (Table 3). There was a difference in the number of CTC between adenocarcinoma and squamous cell carcinoma $(Z=-2.334, P=0.02)$, but no difference between adenocarcinoma and small cell carcinoma $(\mathrm{Z}=-1.435, \mathrm{P}=0.151)$ and between squamous cell carcinoma and small cell carcinoma $(\mathrm{Z}=-1.635, \mathrm{P}=0.102)$.

\section{Relationship between CTC threshold of different ploidy of chromosome 8 and PFS or OS}

We investigated the correlation of CTC counts at different ploidy levels of chromosome 8 with first-line response (PFS) and prognosis (OS) of patients (Table 4). As a result, there were 0 (19 cases), 1 (3 cases), 2 (1 case), and 6 (1 case) monomeric CTC in the peripheral blood of 24 patients 
Table 2 Relationship between positive rate of CTC detection and clinical characteristics of patients

\begin{tabular}{|c|c|c|c|}
\hline Clinical characteristics & $\begin{array}{l}\text { CTC cases } \\
(n=24), n(\%)\end{array}$ & $\chi^{2}$ value & $P$ value \\
\hline \multicolumn{4}{|l|}{ Sex } \\
\hline Male & 16 [15] & 0.522 & 0.470 \\
\hline Female & $8[8]$ & & \\
\hline \multicolumn{4}{|l|}{ Age (years) } \\
\hline$>60$ & 16 [16] & 2.087 & 0.149 \\
\hline$\leq 60$ & $8[7]$ & & \\
\hline \multicolumn{4}{|l|}{ History of smoking } \\
\hline Yes & $12[11]$ & 6.020 & 0.014 \\
\hline No & $12[12]$ & & \\
\hline \multicolumn{4}{|l|}{ ECOG score } \\
\hline $0-1$ & $15[14]$ & 0.626 & 0.429 \\
\hline$\geq 2$ & 9 [9] & & \\
\hline \multicolumn{4}{|l|}{ Pathological type } \\
\hline Adenocarcinoma & 17 [16] & 0.430 & 0.807 \\
\hline Squamous carcinoma & $4[4]$ & & \\
\hline SCLC & $3[3]$ & & \\
\hline \multicolumn{4}{|l|}{ Serum CEA } \\
\hline Normal & $8[8]$ & 0.522 & 0.470 \\
\hline Increased & 16 [15] & & \\
\hline
\end{tabular}

CEA, carcinoembryonic antigen; CTC, circulating tumor cells; ECOG, Eastern Cooperative Oncology Group; SCLC, small cell lung cancer.

before treatment. To determine the threshold of aneuploid CTC for poor outcome, we compared the PFS and OS data of patients with monomeric CTC count $\geq 1$ with those of patients with monomeric CTC count $<1$ and found no statistically significant difference between them $(Z=-0.572$, $\mathrm{P}=0.567 ; \mathrm{Z}=-1.459, \mathrm{P}=0.144)$. Before treatment, there were 0 (19 cases), 1 (4 cases), and 2 (1 case) diploid CTC in peripheral blood, respectively. There was no significant difference in PFS and OS between patients with diploid CTC count $\geq 1$ and those with diploid CTC count $<1$ $(\mathrm{Z}=0.000, \mathrm{P}=1.000 ; \mathrm{Z}=-0.854, \mathrm{P}=0.393)$. There were 0 (10 cases), 1 (6 cases), 2 (4 cases), 3 (2 cases), and 4 (2 cases) triploid CTC in peripheral blood before treatment, respectively. The PFS and OS data of patients with triploid CTC count $\geq 1$ were compared with those of patients with
Table 3 Relationship between numbers of CTC and clinical characteristics of patients

\begin{tabular}{|c|c|c|c|}
\hline Clinical characteristics & CTC number $(n=285)$ & $Z$ value & $P$ value \\
\hline Sex & & -1.137 & 0.255 \\
\hline Male & $7(2.25-11.5)$ & & \\
\hline Female & $8.5(6.25-11.75)$ & & \\
\hline Age (years) & & -0.031 & 0.975 \\
\hline$>60$ & $7.5(3-11.5)$ & & \\
\hline$\leq 60$ & $7(3.25-11.75)$ & & \\
\hline History of smoking & & -0.985 & 0.325 \\
\hline Yes & $8(6.25-10.75)$ & & \\
\hline No & $6(2-13.5)$ & & \\
\hline ECOG score & & -0.15 & 0.881 \\
\hline $0-1$ & $7(3-12.0)$ & & \\
\hline$\geq 2$ & $7(3-10.5)$ & & \\
\hline Pathological type & & -2.334 & 0.029 \\
\hline Adenocarcinoma & $9(6.5-13)$ & & \\
\hline Squamous carcinoma & $2.5(2-4.5)$ & & \\
\hline SCLC & $7(3-)$. & & \\
\hline Serum CEA & & -0.369 & 0.712 \\
\hline Normal & $7(3.5-10.25)$ & & \\
\hline Increased & $7.5(3.25-12)$ & & \\
\hline
\end{tabular}

CEA, carcinoembryonic antigen; CTC, circulating tumor cells; ECOG, Eastern Cooperative Oncology Group; SCLC, small cell lung cancer.

triploid CTC count $<1$. The results showed that there was no significant difference in PFS and OS between them $(\mathrm{Z}=-0.913, \mathrm{P}=0.361 ; \mathrm{Z}=-0.938, \mathrm{P}=0.348)$. The PFS and OS data were compared between patients with triploid CTC count $\geq 2$ and patients with triploid CTC count $<2$. There was no significant difference in PFS and OS between the 2 groups $(\mathrm{Z}=-0.585, \mathrm{P}=0.558 ; \mathrm{Z}=-1.043, \mathrm{P}=0.297)$. There were 0 (12 cases), 1 ( 7 cases), 2 ( 1 case), 3 ( 2 cases), 4 (1 cases), and 5 ( 1 case) tetraploid CTC in peripheral blood before treatment, respectively. Comparison of PFS and OS data between patients with tetraploid CTC count $\geq 1$ and those with tetraploid CTC count $<1$ showed no statistically significant difference in PFS $(Z=-1.568$, $\mathrm{P}=0.117$ ) (Figure 1), but there was significant difference in OS $(\mathrm{Z}=-2.284, \mathrm{P}=0.022)$. Comparing the PFS and OS data of patients with tetraploid CTC count $\geq 2$ and patients 
Table 4 Relationship between CTC threshold of different ploidy of chromosome 8 and PFS or OS

\begin{tabular}{|c|c|c|c|c|c|c|}
\hline $\begin{array}{l}\text { Threshold grouping of different } \\
\text { ploidies }\end{array}$ & \multicolumn{3}{|c|}{ PFS } & \multicolumn{3}{|c|}{ OS } \\
\hline Monomeric CTC $<1$ & $5(3-10)$ & -0.572 & 0.567 & $11(5-19)$ & -1.459 & 0.144 \\
\hline Monomeric CTC $\geq 1$ & $3(2-11)$ & & & $4(2.5-14)$ & & \\
\hline Diploid CTC <1 & $5(3-10)$ & 0.000 & 1.000 & $11(5-19)$ & -0.854 & 0.393 \\
\hline Triploid CTC $<1$ & $4(2.5-7)$ & -0.913 & 0.361 & $8.25(4.5-15.25)$ & -0.938 & 0.348 \\
\hline Triploid CTC $\geq 1$ & $8(2.75-10)$ & & & $11.5(4.75-20)$ & & \\
\hline Triploid CTC <2 & $4.5(3-9.5)$ & -0.585 & 0.558 & $8.25(5-17)$ & -1.043 & 0.297 \\
\hline Triploid CTC $\geq 2$ & $8.5(2.25-9.75)$ & & & $13(5.75-25.5)$ & & \\
\hline Tetraploid CTC <2 & $4(2.25-8)$ & -2.366 & 0.014 & $8.25(4.25-13.5)$ & -2.366 & 0.018 \\
\hline Tetraploid CTC $\geq 2$ & $10(9.25-14.875)$ & & & $22(15.75-27.5)$ & & \\
\hline Polyploid CTC <1 & $7(4.5-8.75)$ & -0.585 & 0.559 & $13(10.5-25.25)$ & -1.319 & 0.187 \\
\hline Polyploid CTC $\geq 1$ & $4.5(2.25-10)$ & & & $8.25(4.25-18)$ & & \\
\hline Polyploid CTC <5 & $5.5(3-9)$ & 0.000 & 1.000 & $11(5-18.25)$ & -0.440 & 0.660 \\
\hline Polyploid CTC $\geq 5$ & $4.5(1.75-10.25)$ & & & $9.75(3.75-18.25)$ & & \\
\hline
\end{tabular}

CTC, circulating tumor cells; OS, overall survival; PFS, progression-free survival.
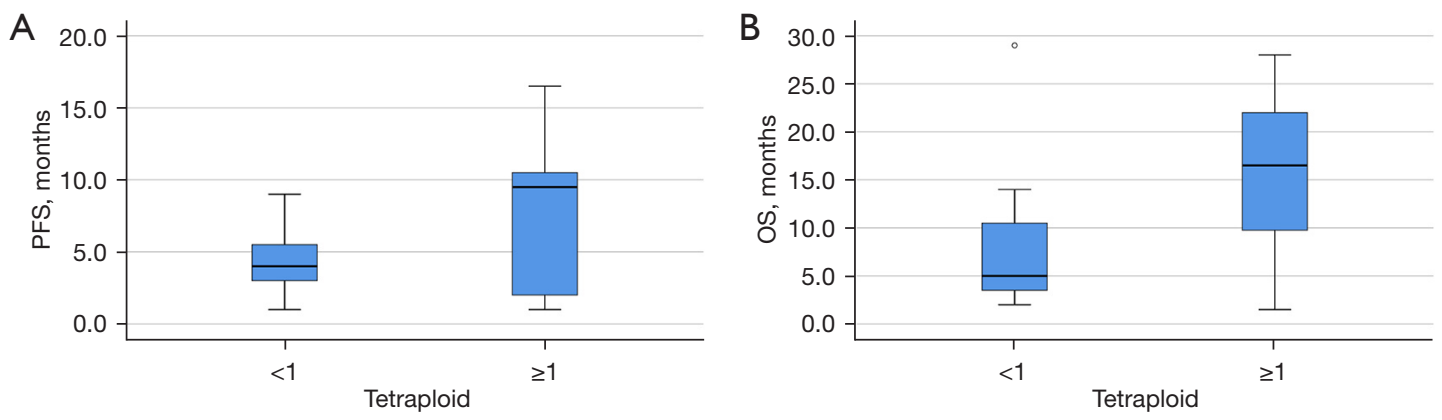

Figure 1 Relationship between the number of tetraploid circulating tumor cells (CTC) $\geq 1$ or $<1$ and the patient's progression-free survival (PFS) or overall survival (OS). (A) Relationship between the number of tetraploid CTC $\geq 1$ or $<1$ and the patient's PFS; (B) relationship between the number of tetraploid CTC $\geq 1$ or $<1$ and the patient's OS.

with tetraploid CTC count $<2$, the results showed that the PFS and OS of the 2 patients were significantly different $(\mathrm{Z}=-2.366, \mathrm{P}=0.014 ; \mathrm{Z}=-2.366, \mathrm{P}=0.018)$ (Figure 2). Before treatment, there were 0 (4 cases), 1-4 (6 cases), $5-9$ (10 cases), and $\geq 10$ (4 cases) polyploid CTCs in peripheral blood, respectively. The PFS and OS data were compared between patients with polyploid CTC count $\geq 1$ and those with polyploid CTC count $<1$. The results showed that there was no significant difference in PFS and OS between the 2 groups $(\mathrm{Z}=-0.585, \mathrm{P}=0.559 ; \mathrm{Z}=-1.319$, $\mathrm{P}=0.187)$. The PFS and OS data of patients with polyploid CTC count $\geq 5$ and patients with polyploid CTC count $<5$ were compared. The results showed that there was no significant difference in PFS and OS between the 2 groups 

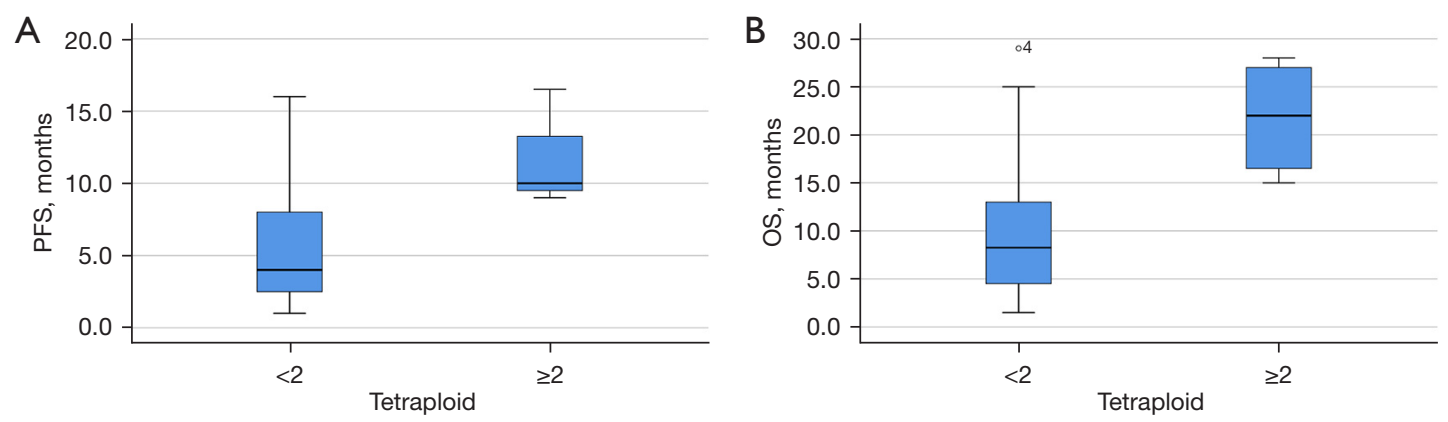

Figure 2 Relationship between the number of tetraploid circulating tumor cells (CTC) $\geq 2$ or $<2$ and the patient's progression-free survival (PFS) or overall survival (OS). (A) Relationship between the number of tetraploid CTC $\geq 2$ or $<2$ and the patient's PFS; (B) relationship between the number of tetraploid CTC $\geq 2$ or $<2$ and the patient's OS.
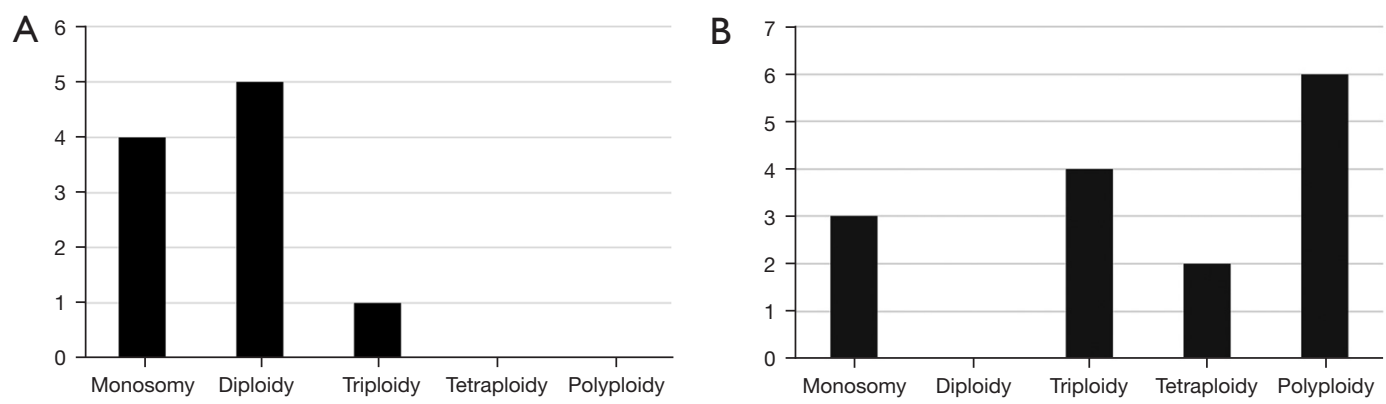

Figure 3 Ploidy ratio of chromosome 8 in Vimentin+/- circulating tumor cells (CTC). (A) Ploidy ratio of chromosome 8 in Vimentin+ CTC; (B) ploidy ratio of chromosome 8 in Vimentin- CTC.

$(\mathrm{Z}=0.000, \mathrm{P}=1.000 ; \mathrm{Z}=-0.440, \mathrm{P}=0.660)$. There was no statistical correlation between CTC count of monosomic, diploid, triploid, and polyploid CTC in PFS and OS $(\mathrm{P}>0.05)$. A tetraploid CTC count of $\geq 2$ or more was an unfavorable predictor of response, it can predict poor PFS in patients with advanced lung cancer. A tetraploid CTC count $\geq 1$ is an unfavorable prognostic predictor of poor OS in patients with advanced lung cancer.

\section{Relationship between the expression of tumor markers and the ploidy of chromosome 8}

Two tumor markers, CK18 and Vimentin, were detected in the present study. All patients were tested for CK18, and only 1 case had both CK18+ CTC (1 triploid) and CK18CTC (1 tetraploid). The remaining 22 patients were CK18CTC. The CK18+ CTC detection rate was $4.35 \%(1 / 23)$. Seven patients (No. B1-B7) were tested for both CK18 and Vimentin; all were negative for CK18, 5 cases $(71.43 \%)$ had both Vimentin+ CTC and Vimentin- CTC, 1 case
(14.29\%) had only Vimentin+ CTC, and the remaining case (14.29\%) had only Vimentin- CTC. The rate of Vimentin+ CTC was $85.71 \%(6 / 7)$. In 6 cases of Vimentin+ CTC, there were 4 cases of monosomy, 5 cases of diploidy, and 1 case of triploidy in chromosome 8. In 6 cases of VimentinCTC, there were 3 cases of monosomy, 4 cases of triploidy, and 2 cases of tetraploidy in chromosome 8 . There were 6 cases of polyploidy, of which 5 cases had $\geq 7$ polyploidy. Therefore, chromosome 8 of Vimentin+ CTC was mostly diploid and monosomic, and absent of polysome. VimentinCTC showed more heteroploid diversity of chromosome 8 , including monosomy, triploid, tetraploid, and polyploid, especially the probability of polyploid was higher (Figure 3).

\section{Tumor marker expression of small cell CTC and chromosome 8 aneuploid}

In the present study, 7 patients (No. B1-B7) had double tumor markers and chromosome 8 aneuploidy. Six patients were found to have small cell $(\leq 5 \mu \mathrm{m}) \mathrm{CTC}$; the total 
number of small cell CTC was 11, the incidence rate was $85.71 \%(6 / 7)$, and the proportion of small cell CTC in total CTC was 11. The tumor marker phenotype of small cell CTC was CK18- and Vimentin+, including 4 monomers (36.36\%), 6 diploids (54.55\%), and 1 trisomy (9.09\%). No tetraploid and polyploid were found.

\section{$C T M$}

CTM ( $\geq 2$ CTC) were found in 2 of 7 patients (No. B1B7), with an incidence of $28.57 \%$ (2/7). The total number of CTM was 2, and the tumor marker phenotypes were CK18- and Vimentin+ and CK18- and Vimentin-, respectively. The 2 cases were IVB adenocarcinoma patients, and serum CEA was significantly increased. They were 33.80 and $57.54 \mathrm{ng} / \mathrm{mL}$, respectively, while the CEA of the other 5 patients was normal or slightly elevated. Patient B4 was treated with first-generation EGFRi because of exon 19 deletions. However, PFS was only 1 month and OS was 2 months. Patient B6 was treated with whole brain radiotherapy because of brain metastasis. PFS was 3 months and OS was 4 months.

\section{Discussion}

At the end of the 19th century, Steven Paget published his "seed and soil" hypothesis of tumor metastasis. It is undeniable that the number of CTC in peripheral blood is very small, sometimes it is difficult to obtain CTC in some patients. In the process of continuous adaptation to the microenvironment of peripheral blood, some CTC undergo EMT. Some show apoptosis or reduced antigen expression (22). To overcome the difficulty of CTC detection caused by these problems, in 2018, Ye et al. studied the clinical significance of aneuploidy of CTC in patients with various malignant tumors (6). A total of 594 blood samples from 479 cases of 19 different cancers and 30 healthy samples were collected for CTC detection and analysis by SEiFISH. The results of the study show that no positive CTC was found in all 30 healthy samples. In the diagnosis of malignant tumors, the overall positive rate of CTC was $89 \%(75-100 \%)$. The results were consistent with the positive rate of CTC detection in our study (95.83\%).

Other studies have shown that an average of $11,5,8$, and 4 CTs per $7.5 \mathrm{~mL}$ of blood were observed in lung, liver, kidney, and colorectal cancers, respectively $(6,25-28)$. In the present study, the average number of CTC detected per $6 \mathrm{~mL}$ blood for each case was 12.4 (285/23), which was similar to the results of previous studies, but slightly better. In addition, more than $95 \%$ of the positive rate was also closely related to the clinical stage and pathological type of the patients enrolled in the present study. All patients were stage IV, except one who was stage IIIB; CTC is more easily detected in patients with metastasis. On the contrary, if a patient cannot complete the clinical staging evaluation, CTC measurement can also effectively predict whether the patient has metastasis, which has certain prognostic value for patients. There are patients with different pathological types: adenocarcinoma $70.83 \%$ (17 cases), small cell carcinoma $12.5 \%$ (3 cases), squamous cell carcinoma $16.67 \%$ (4 cases). It is well known that adenocarcinoma and small cell lung cancer are more likely to metastasize, and the proportion of adenocarcinoma and small cell lung cancer patients in the present study was high (83.33\%); all had advanced lung cancer. Due to the high sensitivity of SE-iFISH, we obtained a high positive rate of $95.83 \%$ for the CTC detection results.

There was no significant correlation between the positive rate of CTC and the clinical characteristics of lung cancer patients, including sex, age, ECOG score, histopathological type, and serum CEA. The results were basically consistent with those of other clinical studies sex, age, histopathological type. However, some other studies noted that the detection rate of CTC in patients with lung cancer in clinical stage III-IV was higher than that in patients with lung cancer in clinical stage I-II (29-31), but we did not find a significant correlation between the detection rate of CTC and the clinical stage of lung cancer in the present study. The possible reason for this result is that the number of patients enrolled in the present study was low, and most patients had stage IV metastatic lung cancer (only 1 case had stage IIIB); there was a lack of early-stage (stages I and II) and stage IIIA patients. However, the results of the present study showed that there was a significant difference between the detection rate of CTC and smoking status $(\mathrm{P}=0.014)$; that is, the detection rate of CTC in patients who smoked was lower than that in non-smoking patients. Only 1 patient in the whole sample was negative for CTC, and this patient had a history of smoking, so this conclusion needs to be further confirmed.

We also found that there was no significant correlation between the number of CTC and the clinical characteristics of patients (including sex, age, ECOG score, and serum CEA), but there was a certain correlation between the number of CTC and the histopathological type of patients. There was a difference in the number of CTC between 
adenocarcinoma and squamous cell carcinoma $(\mathrm{P}=0.02)$, but there was no difference between adenocarcinoma and small cell carcinoma $(\mathrm{P}=0.151)$ or between squamous cell carcinoma and small cell cancer $(\mathrm{P}=0.102)$. We can conclude that the 3 main pathological types of lung cancer (adenocarcinoma, squamous cell carcinoma, small cell carcinoma) have no significant difference in the detection rate of CTC in peripheral blood, but there are some differences in the number of CTC. The difference between adenocarcinoma and squamous cell carcinoma was statistically significant. In NSCLC, it has been found that CTC count is correlated with tumor metastasis $(15,32)$. While adenocarcinoma is a pathological type prone to hematogenous metastasis, squamous cell carcinoma is relatively inert, mostly it grows and develops slowly. It is not easy to invade and metastasize early. So, it is not difficult to understand, compared with squamous cell carcinoma, peripheral blood CTC count of adenocarcinoma patients is more. Small cell lung cancer has the highest degree of malignancy and is most likely to undergo hematogenous metastasis and dissemination in the early stage. However, there was no significant difference in CTC count between small cell carcinoma and adenocarcinoma, and between small cell carcinoma and squamous cell carcinoma, which might be due to the small number of cases of small cell cancer and squamous cell carcinoma analyzed in the present study. There were only 3 cases of small cell carcinoma and 4 cases of squamous cell carcinoma. The median CTC counts (25-75\% spacing) are $9(6.5-13)$ in adenocarcinoma, 2.5 (2-4.5) in squamous cell carcinoma, 7 (3-.) in small cell carcinoma. If the sample size is increased, it is expected that there might be some statistical difference in CTC count between small cell carcinoma and squamous cell carcinoma.

In the present study, SE-iFISH was used to detect the expression of CK18 and Vimentin on the surface of lung cancer cells in peripheral blood, and was combined with the ploidy of CEP 8 to determine CTC. Of the 23 patients in whom CTC were detected, only 1 CK18+ triploid CTC on chromosome 8 was detected in 1 patient, and in the remaining 22 patients, all CTC were CK18-. The positive rate of CK18+ CTC was $4.35 \%$ (1/23). Five of 7 patients had Vimentin+ CTC and Vimentin- CTC simultaneously, only 1 had Vimentin+ CTC, and the others only had Vimentin- CTC; the detection rate of Vimentin+ CTC was $85.71 \%(6 / 7)$. It is noteworthy that chromosome 8 of Vimentin+ CTC is mostly diploid and monosomic. Vimentin- CTC showed more heteroploid diversity of chromosome 8 , including monosomy, triploid, tetraploid, and polyploid, especially polyploid was commonly seen. These results showed the complexity and complementarity of the expression of CTC surface protein markers and chromosome 8 aneuploidy in the peripheral blood of patients with advanced lung cancer, and the detection sensitivity of chromosome 8 aneuploidy was highest, reaching $100 \%$. Vimentin+ was the second highest at $85.71 \%$, while CK18+ was the worst at $4.35 \%$.

Small cell $(\leq 5 \mu \mathrm{m})$ CTC were detected in 6 of 7 patients (85.71\%). Another method for enriching CTC is isolation by size of epithelial tumor cells (ISET), which can only capture CTC with a larger diameter than the filter pore $(>8 \mu \mathrm{m})$ from the blood sample and is independent of the surface antigen of CTC. However, there is also a small number of CTC with a smaller diameter in peripheral blood. This method is easy to lose this part of small-size CTC, because the CTC is similar to or even smaller than blood cells. In the present study, Vimentin expression and CEP 8 aneuploid were used to detect $85.71 \%(6 / 7)$ of patients with primary lung cancer who had small cell CTC in peripheral blood, which avoided the missed detection of small cell CTC $\leq 5 \mu \mathrm{m}$.

Therefore, the SE-iFISH platform improves the sensitivity of CTC detection, and avoids the following limitations to miss detection, such as tumor heterogeneity, CK downregulation or deletion, and small size.

Chromosome aneuploidy exists in various tumor cells. Many studies have shown that aneuploid tumor cells are associated with poor prognosis. Polyploid tumor cells are genetically unstable and not sensitive to radiotherapy and chemotherapy. It is generally believed that the prognosis of patients with polyploid subcloned tumor cells is worse than other heteroploid patients (33).

In the present study, 23 of 24 patients were positive for CTC in peripheral blood, and all were detected with chromosome 8 heteroploid CTC; the ploidy of chromosome 8 was obviously heterogeneous. The number of polyploid CTC was greatest, followed by triploid and tetraploid CTC. We compared the correlation between the cut-off value of different ploidy numbers of chromosome 8 and PFS of the first-line treatment or OS to find a reference method to predict the efficacy and poor prognosis. The results showed that a tetraploid CTC count $\geq 2$ was an unfavorable predictor of response; it can predict poor PFS in patients with advanced lung cancer. A tetraploid CTC count $\geq 1$ is an unfavorable prognostic predictor of poor OS in patients with advanced lung cancer. It is suggested that a quantitative analysis of chromosome 8 tetraploid in 
CTC by SE-iFISH may be a useful tool for evaluating the therapeutic effect and determining the prognosis.

Previously published studies (34) have found that lung cancer metastasis is likely to have occurred in the early stage of lung cancer, and there is dissemination of lung cancer cells. A large number of CTC aggregate and form CTM with a high metastatic potential; this stage is called micrometastasis and is also known as occult metastasis. Therefore, CTM has stronger ability of invasion and metastasis than CTC. In the present study, CTM was found in 2 of 7 lung cancer patients (28.57\%). Both cases were stage IVB adenocarcinoma, and the serum CEA levels were 33.80 and $57.54 \mathrm{ng} / \mathrm{mL}$, respectively. After first-line treatment, the PFS was only 1 and 3 months, respectively, and the OS was 2 and 4 months, respectively. It is suggested that the presence of CTM in peripheral blood may predict the later clinical stage, the worse therapeutic effect, and poor prognosis of lung cancer patients.

The findings of the present study confirm that SE-iFISH can effectively enrich CTC in the peripheral blood of patients with primary lung cancer. According to the specific needs of clinical or research, the immunofluorescence staining of freely selected tumor biomarkers is combined with chromosome FISH to further improve the sensitivity and specificity of CTC detection. CTC effectively detected by SE-iFISH can be used as preliminary predictors of immediate efficacy and prognosis of patients with metastatic primary lung cancer. Quantification of chromosome 8 aneuploidy also shows potential as a predictive biomarker for disease progression and prognosis. Due to the small sample size, there are still some limitations in the present study, which need to be verified using a larger sample.

We believe that the development of stable, sensitive and specific detection methods is the key to carry out CTC related research. In the future, CTC detection methods are not limited to detection, but more and more focus on capturing live CTC cells. Through their characterization at the cellular and molecular levels, we can obtain information related to tumor metastasis and recurrence, drug tolerance and so on. And this field will still be a research hotspot.

\section{Conclusions}

SE-iFISH has a high detection rate of CTC in the peripheral blood of patients with metastatic primary lung cancer, and it can identify small cell CTC. Tetraploid CTC count $\geq 2$ can predict poor PFS in patients with advanced lung cancer. Tetraploid CTC count $\geq 1$ can predict poor OS in patients with advanced lung cancer. CTM can predict poor prognosis in patients with lung cancer.

\section{Acknowledgments}

Funding: This work was supported by Clinical Medicine Science and Technology Special Project of Jiangsu Province-New Clinical Diagnosis and Treatment Technology Tackling (No. BL2012054).

\section{Footnote}

Reporting Checklist: The authors have completed the REMARK reporting checklist. Available at https://dx.doi. org/10.21037/atm-21-6346

Data Sharing Statement: Available at https://dx.doi. org/10.21037/atm-21-6346

Conflicts of Interest: All authors have completed the ICMJE uniform disclosure form (available at https://dx.doi. org/10.21037/atm-21-6346). The authors have no conflicts of interest to declare.

Ethical Statement: The authors are accountable for all aspects of the work in ensuring that questions related to the accuracy or integrity of any part of the work are appropriately investigated and resolved. The study was conducted in accordance with the Declaration of Helsinki (as revised in 2013) and approved by the Institutional Ethical Committee of Northern Jiangsu People's Hospital (No. 2013010). All patients provided signed informed consent.

Open Access Statement: This is an Open Access article distributed in accordance with the Creative Commons Attribution-NonCommercial-NoDerivs 4.0 International License (CC BY-NC-ND 4.0), which permits the noncommercial replication and distribution of the article with the strict proviso that no changes or edits are made and the original work is properly cited (including links to both the formal publication through the relevant DOI and the license). See: https://creativecommons.org/licenses/by-nc-nd/4.0/.

\section{References}

1. Holdenrieder S. Biomarkers along the continuum of care in lung cancer. Scand J Clin Lab Invest Suppl 2016;245:S40-5. 
2. Tufman A, Huber RM. Biological markers in lung cancer: A clinician's perspective. Cancer Biomark 2010;6:123-35.

3. Tayoun T, Faugeroux V, Oulhen M, et al. CTC-Derived Models: A Window into the Seeding Capacity of Circulating Tumor Cells (CTCs). Cells 2019;8:1145.

4. Batth IS, Mitra A, Rood S, et al. CTC analysis: an update on technological progress. Transl Res 2019;212:14-25.

5. Chudasama DY, Freydina DV, Freidin MB, et al. Inertia based microfluidic capture and characterisation of circulating tumour cells for the diagnosis of lung cancer. Ann Transl Med 2016;4:480.

6. Ye Z, Ding Y, Chen Z, et al. Detecting and phenotyping of aneuploid circulating tumor cells in patients with various malignancies. Cancer Biol Ther 2019;20:546-51.

7. Li J, Ai Y, Wang L, et al. Targeted drug delivery to circulating tumor cells via platelet membranefunctionalized particles. Biomaterials 2016;76:52-65.

8. Lin PP. Aneuploid CTC and CEC. Diagnostics (Basel) 2018;8:26.

9. Lin PP. Integrated EpCAM-independent subtraction enrichment and iFISH strategies to detect and classify disseminated and circulating tumors cells. Clin Transl Med 2015;4:38.

10. Wu C, Hao H, Li L, et al. Preliminary investigation of the clinical significance of detecting circulating tumor cells enriched from lung cancer patients. J Thorac Oncol 2009;4:30-6.

11. Rao PH, Zhao S, Zhao YJ, et al. Coamplification of Myc/Pvt1 and homozygous deletion of Nlrp1 locus are frequent genetics changes in mouse osteosarcoma. Genes Chromosomes Cancer 2015;54:796-808.

12. Mettu RK, Wan YW, Habermann JK, et al. A 12-gene genomic instability signature predicts clinical outcomes in multiple cancer types. Int J Biol Markers 2010;25:219-28.

13. Kronenwett U, Huwendiek S, Ostring C, et al. Improved grading of breast adenocarcinomas based on genomic instability. Cancer Res 2004;64:904-9.

14. Sato H, Uzawa N, Takahashi K, et al. Prognostic utility of chromosomal instability detected by fluorescence in situ hybridization in fine-needle aspirates from oral squamous cell carcinomas. BMC Cancer 2010;10:182.

15. Yu JH, Wang D, Jin L, et al. Utility of circulating tumor cells in stage II colorectal cancer patients undergoing curative resection. Transl Cancer Res 2020;9:1487-94.

16. Chen Y, Yang Z, Wang Y, et al. Karyotyping of circulating tumor cells for predicting chemotherapeutic sensitivity and efficacy in patients with esophageal cancer. BMC Cancer 2019;19:651.
17. Gao Y, Zhu Y, Zhang Z, et al. Clinical significance of pancreatic circulating tumor cells using combined negative enrichment and immunostaining-fluorescence in situ hybridization. J Exp Clin Cancer Res 2016;35:66.

18. Li Y, Zhang X, Gong J, et al. Aneuploidy of chromosome 8 in circulating tumor cells correlates with prognosis in patients with advanced gastric cancer. Chin J Cancer Res 2016;28:579-88.

19. Wadhwa N, Mathew BB, Jatawa SK, et al. Genetic instability in urinary bladder cancer: An evolving hallmark. J Postgrad Med 2013;59:284-8.

20. Enane FO, Shuen WH, Gu X, et al. GATA4 loss of function in liver cancer impedes precursor to hepatocyte transition. J Clin Invest 2017;127:3527-42.

21. Wu W, Zhang Z, Gao XH, et al. Clinical significance of detecting circulating tumor cells in colorectal cancer using subtraction enrichment and immunostainingfluorescence in situ hybridization (SE-iFISH). Oncotarget 2017;8:21639-49.

22. Ge F, Zhang H, Wang DD, et al. Enhanced detection and comprehensive in situ phenotypic characterization of circulating and disseminated heteroploid epithelial and glioma tumor cells. Oncotarget 2015;6:27049-64.

23. Zhang Y, Li J, Wang L, et al. Clinical significance of detecting circulating tumor cells in patients with esophageal squamous cell carcinoma by EpCAM independent enrichment and immunostaining fluorescence in situ hybridization. Mol Med Rep 2019;20:1551-60.

24. Gao J, Qin W, Kang P, et al. Up-regulated LINC00261 predicts a poor prognosis and promotes a metastasis by EMT process in cholangiocarcinoma. Pathol Res Pract 2020;216:152733.

25. Wei T, Zhu D, Yang Y, et al. The application of nanoenrichment in CTC detection and the clinical significance of CTCs in non-small cell lung cancer (NSCLC) treatment. PLoS One 2019;14:e0219129.

26. Gradilone A, Iacovelli R, Cortesi E, et al. Circulating tumor cells and "suspicious objects" evaluated through CellSearch ${ }^{\circledR}$ in metastatic renal cell carcinoma. Anticancer Res 2011;31:4219-21.

27. Aranda E, Viéitez JM, Gómez-España A, et al. FOLFOXIRI plus bevacizumab versus FOLFOX plus bevacizumab for patients with metastatic colorectal cancer and $\geq 3$ circulating tumour cells: the randomised phase III VISNÚ-1 trial. ESMO Open 2020;5:e000944.

28. Mendelaar PAJ, Kraan J, Van M, et al. Defining the dimensions of circulating tumor cells in a large series of breast, prostate, colon, and bladder cancer patients. Mol 
Oncol 2021;15:116-25.

29. Ding C, Zhou X, Xu C, et al. Circulating tumor cell levels and carcinoembryonic antigen: An improved diagnostic method for lung adenocarcinoma. Thorac Cancer 2018;9:1413-20.

30. Wang SQ, Shuai ZF, Zhang XJ, et al. Detection of CTCs and CSCs in the staging and metastasis of nonsmall cell lung cancer based on microfluidic chip and the diagnostic significance. Eur Rev Med Pharmacol Sci 2020;24:9487-96.

31. Liang N, Liu L, Li P, et al. Efficient isolation and quantification of circulating tumor cells in non-small cell

Cite this article as: Yao H, Wang Z, Yang J, Xu X, Song Y. Simultaneous in situ detection of protein expression of multiple tumor markers of circulating tumor cells and heteroploid of chromosome 8 in primary lung cancer. Ann Transl Med 2021;9(24):1772. doi: 10.21037/atm-21-6346 lung cancer patients using peptide-functionalized magnetic nanoparticles. J Thorac Dis 2020;12:4262-73.

32. Shishido SN, Carlsson A, Nieva J, et al. Circulating tumor cells as a response monitor in stage IV non-small cell lung cancer. J Transl Med 2019;17:294.

33. Shimono J, Miyoshi H, Kiyasu J, et al. Clinicopathological analysis of polyploid diffuse large B-cell lymphoma. PLoS One 2018;13:e0194525.

34. Tian Y, He Y, Li X, et al. Novel nomograms to predict lymph node metastasis and distant metastasis in resected patients with early-stage non-small cell lung cancer. Ann Palliat Med 2021;10:2548-66. 